\title{
Research Based on the Frequency Hopping Wireless Communication Networks
}

\author{
Shuang Wu \\ College of Information Engineering, Nanchang Hangkong \\ University, Jiang xi, Nan Chang, China \\ e-mail:shuang12wu@163.com
}

\begin{abstract}
Wireless networking is becoming more and more popular, because of the usage wireless communication technology. Therefore, it will inevitably be affected by multipath fading, interference tracking, interception and other effects in the communication process. The RF signal frequency bandwidth after expanding spectrum communication may be several times or even several thousands of times as strong as the original signal frequency bandwidth. With strong anti-jamming, anti-fading, anti-interception capability, it also has the advantage of multi-site networking and so on. This paper developed a wide interval of $R S$ codes treatment by modulo nonlinear method, processed by MATLAB simulation, compared the dual-band method, derived that the sequence of modulo nonlinear method is higher in structural discrete structure, anti-interference ability.
\end{abstract}

Keywords-Frequency hopping communication; Wifi; Wide interval frequency hopping sequence; Communications Interference

\section{INTRODUCTION}

Spread spectrum communication uses information-independent and pseudo-random sequence to make the RF signal bandwidth is far more than the information signal (baseband signal) band width, the principle is using of a pseudo-random sequence to make the carrier frequency in a relatively wide expansion, so that signal can be transmitted invisibly within the bandwidth. Using frequency hopping communication system, adjusting the pseudo-random code and hopping pattern, increasing anti-interference ability of communication systems provide a good environment for communication and ad hoc network of wireless sensors. This paper developed a wide interval of RS codes treatment by modulo nonlinear method, processed by MATLAB simulation, compared the dual-band method, derived that the sequence of modulo nonlinear method is higher in structural discrete structure, anti-interference ability.

\section{DESIGN OF WIDE INTERVAL FREQUENCY HOPPING SEQUENCE.}

\section{A. The mathematical model of frequency hopping communication.}

In1948 C.E.Shannon published paper entitled "A Mathematical Theory of Communication", laid the foundation for the theory of spread spectrum communication. The Shannon formula is as follows:

\author{
Ke Zhao \\ College of Information Engineering, Nanchang Hangkong \\ University, Jiang xi, Nan Chang, China \\ e-mail: zhaoke6805@126.com
}

$$
C=B \times \log _{2}\left(1+\frac{S}{N}\right)
$$

Channel capacity is represented by $\mathrm{C}$, channel bandwidth is represented by $\mathrm{B}$, signal power is represented by $\mathrm{S}$, white noise power is represented by $\mathrm{N}$.

Assuming $p(t)$ is the carrier signal combined by the local carrier frequency and hopping pattern, it can be expressed as:

$$
p(\mathrm{t})=\cos \left[\left(\omega_{0}+\mathrm{n} \omega_{\Delta}\right) \mathrm{t}+\varphi_{\mathrm{n}}\right]
$$

$\mathrm{RF}$ center frequency is represented by $\omega_{0}$, frequency hopping interval is represented by $\omega_{\Delta}$, initial phase for each hop is represented by $\varphi_{\mathrm{n}}, \mathrm{n}$ is a uniformly distributed random variable.

Thus, the information $\mathrm{M}$ after modulation forms baseband waveform $m(t)$, spread spectrum carrier $p(t)$ carrying the baseband waveform is the transmitter frequency hopping signal $\mathrm{Si}(\mathrm{t})$.

$$
\mathrm{S}_{\mathrm{i}}(\mathrm{t})=\mathrm{m}(\mathrm{t}) \times p(\mathrm{t})=\mathrm{m}(\mathrm{t}) \times \cos \left[\left(\omega_{0}+\omega_{\Delta}\right) \mathrm{t}+\varphi_{\mathrm{n}}\right](3)
$$

In the process of signal transmission, the channel exists noise signal $\mathrm{n}(\mathrm{t})$, other hopping signal $\mathrm{Sj}(\mathrm{t})$ and interference $\mathrm{J}(\mathrm{n})$, then the receiver receives a signal $\mathrm{Sr}(\mathrm{t})$ can be expressed as:

$\mathrm{S}_{\mathrm{r}}(\mathrm{t})=\mathrm{S}_{\mathrm{i}}(\mathrm{t})+\sum_{j=2}^{\mathrm{k}} \mathrm{S}_{\mathrm{j}}(\mathrm{t})+\mathrm{n}(\mathrm{t})+\mathrm{J}(\mathrm{t})(4)$

At the receiving end, hopping carrier signal generated by local frequency synthesizer and a received signal $\operatorname{Sr}(\mathrm{t})$ despread, forming a despreaded signal $\mathrm{Sp}(\mathrm{t})$ :

$\mathrm{S}_{\mathrm{P}}(\mathrm{t})=\left[\mathrm{S}_{\mathrm{i}}(\mathrm{t})+\sum_{j=2}^{2 \mathrm{k}} \mathrm{S}_{\mathrm{j}}(\mathrm{t})+\mathrm{n}(\mathrm{t})+\mathrm{J}(\mathrm{t})\right] \cos \left[\left(\omega_{\mathrm{r}}+\mathrm{n} \omega_{\Delta}\right) \mathrm{t}+\varphi_{\mathrm{r}}\right](5)$

Center frequency is represented by $\omega_{\mathrm{r}}$, initial phase is represented by $\varphi_{\mathrm{r}}$. Among them, the difference between $\omega_{\mathrm{r}}$ and $\omega_{0}$ is an intermediate frequency. If $\mathrm{FH}$ synchronization of both sending and receiving has been achieved, there is: 


$$
\begin{gathered}
S_{p}(t)=\frac{1}{2} m(t) \times\left\{\cos \left(\omega_{i}(t)+\varphi_{i}\right)+\cos \left[\left(\omega_{0}+\omega_{r}+2 n \omega_{\Delta}\right) t+\varphi_{n}+\varphi_{r}\right]\right\}+ \\
{\left[\sum_{j=2}^{k} S_{j}(t)+n(t)+J(t)\right] \times \cos \left[\left(\omega_{r}+n \omega_{\Delta}\right) t+\varphi_{r}\right]}
\end{gathered}
$$

$\varphi_{i}=\varphi_{r}-\varphi_{n}, \omega_{i}=\omega_{r}-\omega_{0}$, This equation shows that: As for the communication has $\mathrm{FH}$ synchronization, the receiver in each dwell time $n T \leq t \leq(n+1) T$ outputs an intermediate frequency, then the intermediate frequency signal filters to be useful signal $\mathrm{Sd}(\mathrm{t})$ :

$$
S_{d}(t)=m(t) \times \cos \left(\omega_{i} t+\varphi_{i}\right)
$$

The $\mathrm{Sd}(\mathrm{t})$ is brought to a demodulator, by demodulation, restore information in the modulation signal $\mathrm{m}(\mathrm{t})$ and goes through the channel decoder decoding, recover the originally transmitted message M. For noise, other hopping communication transmitter signal and interfering signals, due to the local carrier cannot generate fixed intermediate frequency after mixing with local hopping carrier wave, and therefore the signal through the mixer would filter the other signal by Intermediate frequency bandpass filter, which effectively avoids interference of other signals.

\section{$B$. Wide interval sequence of $R-S$ code constructed by nonlinear modulo}

The most widely used is the dual-band method, by dual-band treatment, the sequence is capable of widen hopping pattern, however, this method will result in some frequencies are ignored, reducing the number of hops frequently point in the project, but also not a good solution to the problem of collisions of sequence. Therefore, this paper designed a nonlinear modulo method to construct wide interval frequency hopping sequence, the proposed method can effectively avoid the problem of reducing the frequency points, while the wide interval processing sequence can achieve good results. According to the method, RS code will be generated once be set:

$S_{1=\{1,2,4,8,16,32,3,6,12,24,48,}$ $35,5,10,20,40,19,38,15,30,60,59,53$, $41,17,34,7,14,28,56,51,37,9,18,36$, $11,22,44,27,54,47,29,58,55,45,25,50$, $39,13,26,52,43,21,42,23,46,31,62,63$, $61,57,49,33\}$

Nonlinear modulo method to construct a wide interval sequence works as follows:

(1) Generating method in accordance with the non-systematic RS sequence, q represents RS sequence period.

(2) Assuming frequency width is d,when $\left|f_{i+1}-f_{i}\right|>d \quad, \quad f_{i+1}=f_{i+1} \quad$. Otherwise $f_{i+1}=f_{i+1}+q+f_{i} \bmod d, q=p^{r}$

(3) Taking the sequence is end to end into account, therefore, when the interval bettween last frequency point and initial frequency point is not $\mathrm{d}$, process $f_{q}=f_{q}+d$.

(4) Replaces the original frequency with processed frequency, and the form a new sequence.

By the above method, use the non-linear modulo to present the sequence:

$S_{1}^{\prime}=\{1,67,4,72,16,32,3,71,12,88$, $48,99,5,75,20,40,19,38,15,30,60,123$, $53,106,17,34,7,79,28,56,115,37,9,83$, $36 \quad, 11,87,44,27,54,111,29,58,119$, $45,25,50,103,13,91,52,107,21,42,23$, $46,31,62,127,61,122,49,33\}$

Width between adjacent frequencies diagram shown in Figure 1.
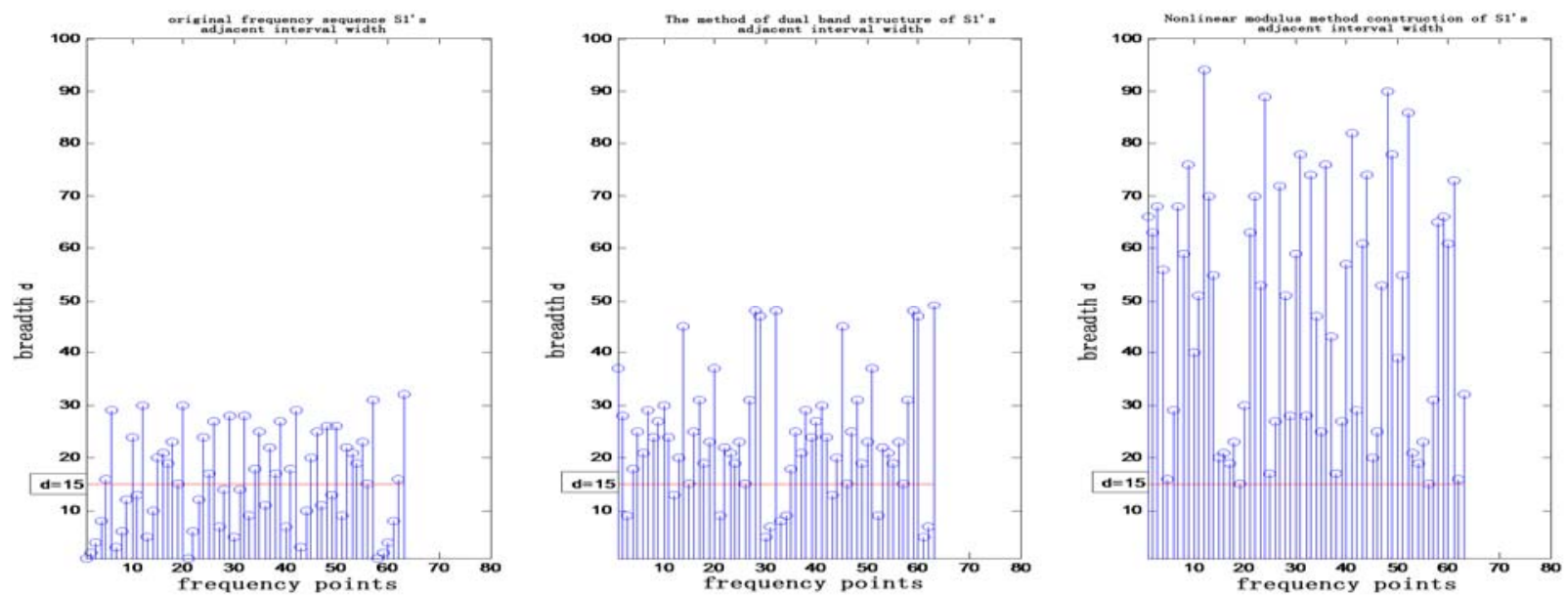

Figure 1. Width between adjacent frequencies

From Figure (2-1) shows that some of the original sequence and the sequence structure of the dual-band method do not satisfy the requirements of inter-wide structure of $d=$
15 , but using the sequence method of nonlinear structure can satisfy their design requirements, and the sequence of nonlinear modulo construction method has high resolution, 
the interval between beginning and end also meet the requirements.

\section{Performance analysis based on wide interval sequence of modulo nonlinear method}

Hopping sequence is used to control the carrier frequency hopping, so the design of hopping sequence to have a direct impact on the ability to resist interference.In general, the Hamming correlation of hopping sequence is one of the important indicators of the hopping sequence. Hamming autocorrelation is to detectet the number of times of collison in a sequence of cycles, after a delay of several hours. Hamming cross-correlation is to detecte the number of times of collision of two sequences of the same length within a sequence period,

In a relatively delay $\tau, L$ is the length of the cycle frequency hopping sequence ,Hamming autocorrelation is:

$$
\begin{gathered}
H_{s_{u} s_{u}}(\tau)=\sum_{i=1}^{L} h\left[s_{u}(j), s_{u}(j+\tau)\right], 1 \leq \tau \leq L \\
h\left[s_{u}(j), s_{u}(j+\tau)\right]= \begin{cases}1 & s_{u}(j)=s_{u}(j+\tau) \\
0 & s_{u}(j) \neq s_{u}(j+\tau)\end{cases}
\end{gathered}
$$

Clearly, in a sequence cycle, the smaller the autocorrelation sequence, indicating that the smaller the number of its collision, the better the ability to resist interference track.
Assuming two hopping sequence length are L, the relative delay in the Hamming cross-correlation is:

$$
\begin{gathered}
H_{S_{u} S_{v}}(\tau)=\sum_{i=1}^{L} h\left[s_{u}(j), s_{v}(j+\tau)\right], 1 \leq \tau \leq L \\
h\left[s_{u}(j), s_{v}(j+\tau)\right]= \begin{cases}1 & s_{u}(j)=s_{v}(j+\tau) \\
0 & s_{u}(j) \neq s_{v}(j+\tau)\end{cases}
\end{gathered}
$$

It can be seen from the definition 2, Hamming correlation is shown in a sequence cycle, the less two frequency hopping sequence collide in relative delay, the less two frequency hopping sequence collide, which means the users of frequence hopping communication are affected less.

We generated RS code through non-systematic approach,we select two ramdom sequences, assuming that the interval between the frequency width $d=15$, according to (8) and (9), we obtain the sequence structure after two periods Hamming autocorrelation and cross correlation. Figure 2 shows a Hamming autocorrelation and cross correlation of nonlinear modulo method constructs a sequence of cycles wide interval.

As can be seen from Fig. (2), the sequence of the Hamming auto-correlation, by calculating the autocorrelation found at 1 (overlap 63 frequencies), other time zero. The cross-correlation with the sequence when the cross-correlation is 1 (overlap 63 frequency points). Figure (3) Shows the diagram in discrete space of sequence.

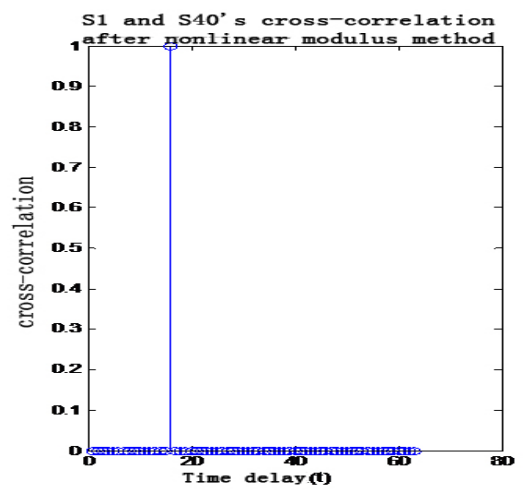

Figure 2. Autocorrelation and cross correlation of nonlinear modulo method

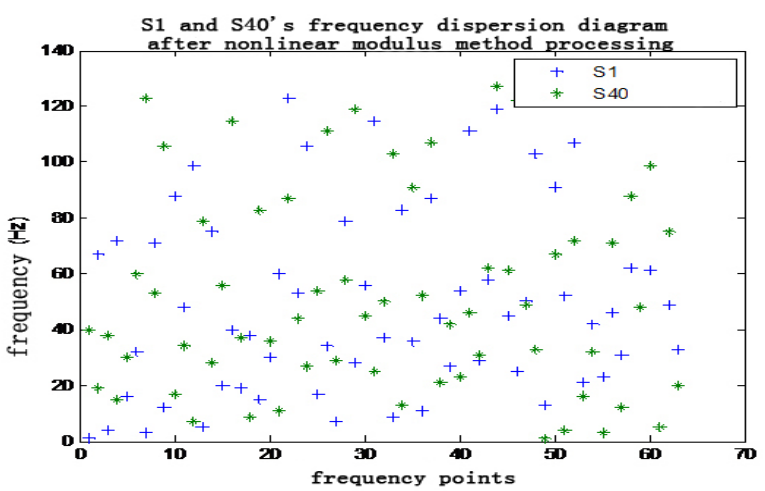

Figure 3. Frequency hopping sequence diacrete diagram of non-linear modulo method 
By studying the autocorrelation and the cross-correlation method of nonlinear modulo method, we found wide interval sequence nonlinear modulo method in the context of the excellent characteristics can expand the width between sequence, and effectively improve the anti-interference
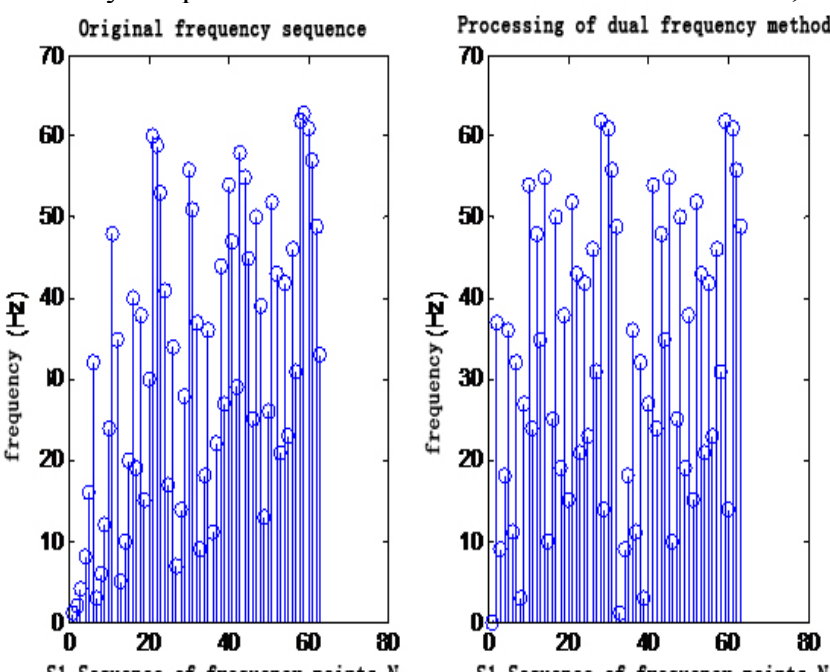

S1 Sequence of frequency points $\mathrm{N}$

1 Sequence of frequency points ability of hopping communications, in the meanwhile, by comparison of Figure (4), the sequence after non-linear modulo method treatment has improved in the frequency width, maximum frequency, and evenly distributed.,compared with that after dual-band treatment.

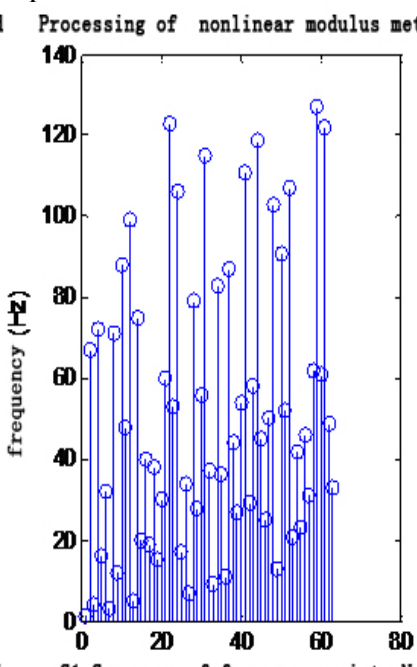

Figure 4. R-S comparison of code frequency sequence before processing and after processing

\section{III.SUMMARY}

This paper designed a nonlinear modulo method for the hopping sequence, which can construct hopping sequence with a wide interval, and comparatively analyze the most commonly used method which is dual-band and found the sequence after nonlinear modulo method treatment has better Hamming autocorrelation and cross correlation and better spatial extent of discrete. Reaching the ability to resist interference.

\section{REFERENCES}

[1] Yang Tian ,Sheng Liyuan ,Zhang Zhanfeng.Design of Chaotic Frequency Hopping Communication System Based on DSPBuilder[J].Computer Measurement \& Control， 2012-20（1） ;
[2] ROMER K, Mattern F, Zurich E. The design space of wireless sensor networks[J]. IEEE Wireless Communications, 2004, 1(16) : 54-61;

[3] Mei Wenhua.frequency hopping communication[M].Beijing : National Defence Industry Press, 2005.4;

[4] B. Majone, F. Viani , E. Filippi, A. Bellin, A. Massa, G. Toller, F. Robol, M. Salucci. Wireless SensorNetwork Deployment for Monitoring Soil Moisture Dynamics at the Field Scale[J]. Procedia Environmental Sciences, 2013, Vol.19, pp.426-435;

[5] Masoud Rezaei, Emad Ebrahimi, Sasan Naseh, Mehdi Mohajerpour. A new 1.4-GHz soil moisture sensor[J]. Measurement, 2012, Vol.45 (06), pp.1723-1728;

[6] Lu Jihua ; Huang Chenfei ; Li Xiangming.A new interleaving method in the adaptive frequency hopping system[J].Journal of Harbin Institute of Technology, 2013, Vol.01 ;

[7] M.S. McCready, M.D. Dukes. Landscape irrigation scheduling efficiency and adequacy by various control technologies[J]. Agricultural Water Management , 2010, Vol.98 (06), pp.697-704. 